\title{
Interspecific movement of the paternal sex ratio chromosome
}

\author{
STEPHEN L. DOBSON* \& MARK A. TANOUYE \\ Department of Environmental Science, Policy and Management, University of California, Berkeley, 131 LSA, \\ Berkeley, CA 94720, U.S.A.
}

\begin{abstract}
Here we examine the potential for interspecific movement of a supernumerary or B chromosome and its subsequent stability. B chromosomes differ from autosomes in that they are nonvital, nonpairing chromosomes which vary in number between conspecific individuals. According to one generally accepted hypothesis, B chromosomes originate from the autosomes of their host. However, previous comparisons of B chromosome and host autosome sequences have suggested an additional evolutionary route: that B chromosomes originating in one species may be subsequently transferred into another species via interspecific hybridization. To examine B chromosome movement, hybrid crosses were utilized to introduce the paternal sex ratio chromosome (PSR) of Nasonia vitripennis into $N$. giraulti and $N$. longicornis. The results demonstrate that these transfers occur at high rates and that the meiotic drive system of PSR continues to function in both species, resulting in the maintenance of PSR at high frequencies. The relevance of these results to origin hypotheses of PSR is discussed here, as are the potential ecological effects of naturally occurring PSR movement and the potential applied uses of the mechanisms of PSR.
\end{abstract}

Keywords: B chromosome, Chalcidoidea, Nasonia giraulti, N. longicornis, $N$. vitripennis, paternal sex ratio chromosome.

\section{Introduction}

B chromosomes occur widely throughout both the plant and animal kingdoms. They are distinct from autosomes by their absence of pairing, variability in number, small size and highly heterochromatic morphology. They also harbour few (if any) genes and typically contribute a small fitness cost to the host organism(Jones \& Rees, 1982; Beukeboom, 1994). This places B chromosomes in the category of 'selfish' genetic elements which replicate and transmit themselves despite being detrimental to their host. To maintain themselves within populations despite their associated genetic load, B chromosomes possess various meiotic drive systems which increase their inheritance to rates above Mendelian predictions (Jones, 1991; Beukeboom, 1994). These varied systems of drive make B chromosomes a unique system in which to study genomic conflict, chromosomal inheritance and chromosomal evolution. One of the most extreme and unique examples

\footnotetext{
*Correspondence and present address: Department of Epidemiology and Public Health, Yale University, 602 LEPH, New Haven, CT 06510, U.S.A. E-mail: stephen.dobson@yale.edu
}

of this drive is found in the paternal sex ratio chromosome (PSR), which enhances its transmission by selfishly manipulating the haplodiploidy of its host Nasonia vitripennis (Hymenoptera; Chalcidoidea). In this haplodiploid system, fertilized eggs develop as diploid females and unfertilized eggs develop as haploid males. However, eggs which are fertilized by sperm harbouring PSR abnormally lose the paternally contributed autosomes, leaving only the maternal complement and PSR. The resulting individuals develop as haploid, PSR males (Werren et al., 1987; Nur et al., 1988; Dobson \& Tanouye, 1996). Thus, matings of PSR males produce all-son broods with the percentage of sons harbouring PSR equivalent to the fertilization rate. The remainder of the broods consist of non-PSR males resulting from unfertilized eggs.

PSR and other B chromosomes are generally believed to originate from the autosomes of their host species (Jones \& Rees, 1982; Beukeboom, 1994). Previously suggested evolutionary hypotheses for the origin of B chromosomes include polysomic events generated by misdivision, asymmetrical translocation, fission or nondisjunction(Jones \& Rees, 
1982). In contrast to this hypothesis, cytological and molecular differences between B chromosomes and the autosomes of their hosts (Beukeboom, 1994) suggest that some B chromosomes may have originated outside their current host. Of particular interest are the reports that several sequences on PSR are more closely related to autosomal sequences of $N$. longicornis and $N$. giraulti than to $N$. vitripennis (Eickbush et al., 1992; McAllister, 1995). One previously proposed hypothesis of the origin of PSR suggests that an interspecific cross may have occurred between a $N$. vitripennis female and a male from another species (Nur et al., 1988; Eickbush et al., 1992; McAllister, 1995). According to this hypothesis, autosomal fragmentation resulted from this cross as a result of Wolbachia-induced cytoplasmic incompatibility (Ryan et al., 1987), and one of these resulting fragments subsequently evolved into what is now PSR.

An alternative origin hypothesis is that PSR evolved outside $N$. vitripennis and was introduced into this species via interspecific hybridization as a functional chromosome (i.e. capable of inducing autosome loss). For an interspecific transfer of this type to occur, there must be an opportunity for interspecific mating, followed by the successful introduction and maintenance of PSR within the recipient species. Studies of mating behaviour and population distributions demonstrate that mating can occur between these Nasonia species under laboratory conditions and may occur naturally (Darling \& Werren, 1990; van den Assem \& Werren, 1994). Hybrid offspring are typically prevented by naturally occurring infections of the Wolbachia microorganism, which cause cytoplasmic incompatibility. However, matings of N. vitripennis and N. giraulti cleared of Wolbachia infection can produce hybrid females when crossed interspecifically (Breeuwer \& Werren, 1990).

This study provides an experimental test of the hypothetical interspecific transfer of PSR. Males harbouring PSR that have been cleared of Wolbachia infection (Dobson \& Tanouye, 1996) are used to introduce PSR into $N$. giraulti and $N$. longicornis. The results demonstrate that PSR can induce autosome loss and is stably transmitted in all three Nasonia species. The ability of the drive system of PSR to function outside N. vitripennis demonstrates that a possible third evolutionary route for PSR should not be excluded: that its ability to induce paternal autosome loss evolved prior to its introduction into $N$. vitripennis. These results also suggest that PSR may continue to function within additional, less related species.

\section{Materials and methods}

Wasp species and strains

Nasonia biology and culturing techniques have been described in detail previously (Whiting, 1967; Beukeboom \& Werren, 1993a). Two N. vitripennis strains were utilized: a Wolbachia-uninfected Leiden Lab II strain (LBw-) and an uninfected strain of males harbouring PSR (PSRw-) (Dobson \& Tanouye, 1996). An infected and uninfected strain of N. giraulti (Breeuwer \& Werren, 1990) and infected N. longicornis (Darling \& Werren, 1990) were also utilized. All wasp strains except PSRwwere maintained by crossing individuals of the same species and Wolbachia infection type. The PSRwstrain was maintained by pair mating with LBwfemales. For maintenance and crossing experiments, Sarcophaga bullata pupae were used as hosts. Two cross types were used for experiments: one male mated with an individual female (pair mating) or with three females (group mating). Both pair and group matings were placed on two hosts. To promote sperm utilization, females were given hosts one day after mating (van den Assem, 1977).

\section{Phenotypic assay}

The fertilization rate for both $N$. giraulti and N. longicornis was determined by observing the percentage of daughters (i.e. $F_{1}$ females) in control crosses of non-PSR males. Because fertilized eggs develop into females and unfertilized eggs into males, the percentage of females is equivalent to the percentage of fertilized eggs. As PSR males are not morphologically distinguishable from non-PSR males, the presence of PSR was determined by pair mating them and observing the sex ratio of their broods. All-son or mostly daughter broods were interpreted as having PSR or non-PSR fathers, respectively. The transmission rate of PSR was determined using a previously developed phenotypic assay (Werren \& van den Assem, 1986); its transmission rate is interpreted as the percentage of sons $\left(F_{1}\right)$ which produce all-son broods $\left(F_{2}\right)$ when pair mated.

\section{PCR amplification}

A PCR assay was used to confirm the presence of the PSR chromosome in wasps. Isolation of DNA and PCR amplification in this assay was identical to that previously described (Dobson \& Tanouye, 1996). In brief, whole wasps were crushed and extracted in 5\% CHELEX 100 resin (BioRad). The 
supernatant ( $1 \mu \mathrm{L})$ was amplified and analysed by electrophoresis. Similar to the previous report, the presence of PSR was indicated by a high molecular weight smear which is absent in non-PSR individuals.

\section{Results}

Introduction of PSR into N. giraulti and N. longicornis

The interspecific transfer of PSR into N. giraulti was accomplished by pair mating PSRw- males with Wolbachia-infected N. giraulti females (Fig. 1). As a control, LBw- males were also crossed with $N$. giraulti females. In PSR crosses, 14/16 crosses (88\%) resulted in all-son broods. The remaining broods consisted of mostly daughters and were assumed to result from non-PSR fathers. This percentage of non-PSR fathers is expected because PSR broods consist of both PSR and non-PSR sons resulting from fertilized and unfertilized eggs, respectively (Werren \& van den Assem, 1986). All of the sons in these broods were of the $N$. giraulti morphological phenotype (Darling \& Werren, 1990). All control crosses $(n=10)$ of non-PSR males yielded broods of mostly daughters. These control cross results are similar to a previous report (Breeuwer \& Werren, 1990) and confirm that the all-son broods resulting from the PSR test crosses did not result from genetic incompatibility or Wolbachia-induced cytoplasmic incompatibility. Instead these all-son broods were caused by the presence of PSR. This demonstrates that PSR continues to induce autosome loss in interspecific crosses introducing it from N. vitripennis into $N$. giraulti eggs. The $N$. giraulti phenotype of the all-son broods demonstrates that PSR caused the loss of the paternal autosomes ( $N$. vitripennis) in these hybrid crosses.

The introduction of PSR into N. longicornis was more complicated because matings with $N$. vitripennis males fail to produce hybrid offspring, regardless of Wolbachia infection (Fig. 1). The cause of this failure to hybridize is likely to result from mate discrimination (van den Assem \& Werren, 1994). Thus, PSR could not be directly introduced from $N$. vitripennis into N. longicornis. However, hybrid offspring are produced in crosses of uninfected $N$. giraulti males with $N$. longicornis females. This allowed the 'double jumping' of PSR into N. longicornis in the present study (Fig. 1). To generate Wolbachia-uninfected N. giraulti sons harbouring PSR, PSRw- males were crossed with uninfected $N$. giraulti females. The resulting sons were then crossed with $N$. longicornis females. The results were similar to those obtained with $N$. giraulti crosses: $82 \%$ of these crosses $(n=17)$ resulted in all-son broods of the $N$. longicornis phenotype. All control crosses $(n=8)$ of uninfected, non-PSR $N$. giraulti
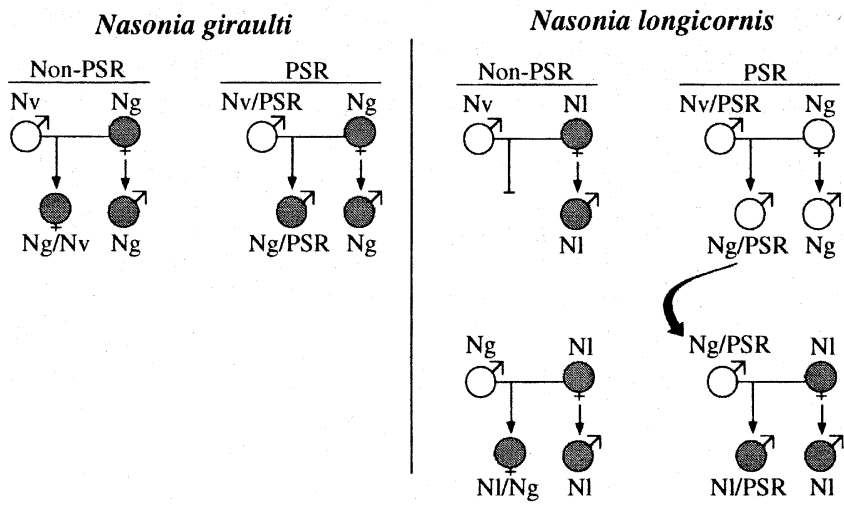

Fig. 1 Diagram of the experimental design allowing the introduction of the paternal sex ratio chromosome (PSR) from Nasonia vitripennis $(\mathrm{Nv})$ into $N$. giraulti $(\mathrm{Ng})$ and N. longicornis $(\mathrm{Nl})$. Maternally transmitted Wolbachia infections are indicated by shaded circles. The presence of PSR in males is indicated adjacent to the species genotype. Nasonia giraulti eggs which are fertilized by Wolbachia-uninfected, non-PSR $N$. vitripennis males produce hybrid female offspring $(\mathrm{Ng} / \mathrm{Nv})$. In these crosses, unfertilized eggs become haploid males $(\mathrm{Ng})$ with their mother's genotype. Because of Wolbachia's maternal route of transmission, all offspring of the infected females are infected with Wolbachia. In the corresponding PSR crosses, N. giraulti eggs are fertilized by uninfected $N$. vitripennis males harbouring PSR. Broods resulting from these latter crosses consist of all-sons of the $N$. giraulti phenotype. Based on the behaviour of PSR in N. vitripennis, fertilized eggs are expected to harbour PSR whereas unfertilized eggs should not (see text). Again, because the mother in these crosses is infected, the offspring also harbour Wolbachia. Matings between $N$. vitripennis males and $N$. longicornis females fail to produce hybrid offspring even if the males are uninfected with Wolbachia. However, uninfected $N$. giraulti males can produce hybrid offspring $(\mathrm{Nl} / \mathrm{Ng})$ when mated with $N$. longicornis. To circumvent the reproductive barrier between $N$. vitripennis and $N$. longicornis, PSR is first introduced into uninfected $N$. giraulti males. Generation of these males is similar to that described above, except that uninfected $N$. vitripennis males harbouring PSR are crossed with uninfected $N$. giraulti females. Because the mother is uninfected, the offspring are also uninfected with Wolbachia. Uninfected $N$. giraulti males harbouring PSR crossed with $N$. longicornis females produce infected, all-son broods of the $N$. longicornis phenotype. Similar to the previous crosses, these all-son broods are expected to include PSR males (fertilized eggs) and non-PSR males (unfertilized eggs). 
males with $N$. longicornis females resulted in mostly daughter broods.

To confirm crossing results suggesting that PSR had been introduced into $N$. giraulti and $N$. longicornis, a PCR detection assay previously developed for N. vitripennis was used (Dobson \& Tanouye, 1996). Males harbouring PSR were maintained for four generations in both $N$. giraulti and $N$. longicornis by randomly crossing males from all-son broods with $N$. giraulti and N. longicornis females, respectively. Males from both $N$. giraulti and $N$. longicornis all-son broods were then PCR assayed. As a negative control, $N$. giraulti and $N$. longicornis males resulting from mostly daughter broods were also tested. The results of one PCR experiment are shown in Fig. 2. With both $N$. giraulti and $N$. longicornis, a majority of males from all-son broods are positive for PSR, whereas none of the control crosses are positive. These results are similar to those observed for PSR in N. vitripennis (Dobson \& Tanouye, 1996) and demonstrate that PSR was successfully introduced into both $N$. giraulti and $N$. longicornis as a result of the interspecific crosses.

\section{Transmission rate of PSR in N. giraulti and N. longicornis}

To examine the ability of PSR to induce paternal autosome loss and to determine its transmission rate in a $N$. giraulti and $N$. longicornis background, $N$. giraulti and N. longicornis males harbouring PSR were pair mated with conspecific females. For comparison, non-PSR males (from mostly daughter broods) were similarly crossed. Nasonia giraulti and N. longicornis females pair mated with non-PSR males produced mostly daughter broods (average 91.2\% and $71.3 \%$, respectively; Fig. 3). This high brood sex ratio is similar to that observed in pair mated N. vitripennis (Werren, 1980, 1983; 1987b). For both $N$. giraulti and $N$. longicornis, the presence of PSR in pair matings caused a dramatic shift in the brood sex ratios. With these PSR pair matings, the majority (Fig. 3) of broods consisted of all sons, whereas a minority were mostly daughter broods. This bimodal distribution is similar to crossing results of PSR in N. vitripennis (Werren \& van den Assem, 1986; Beukeboom \& Werren, 1993b; Dobson \& Tanouye, 1996) and reflects the mixed genotypes (PSR and non-PSR males) of broods resulting from PSR crosses. Crosses of non-PSR males with $N$. giraulti and N. longicornis females demonstrated that females fertilize the majority of their eggs in pair matings. An equivalent majority of males in $N$. giraulti and $N$. longicornis PSR crosses were determined

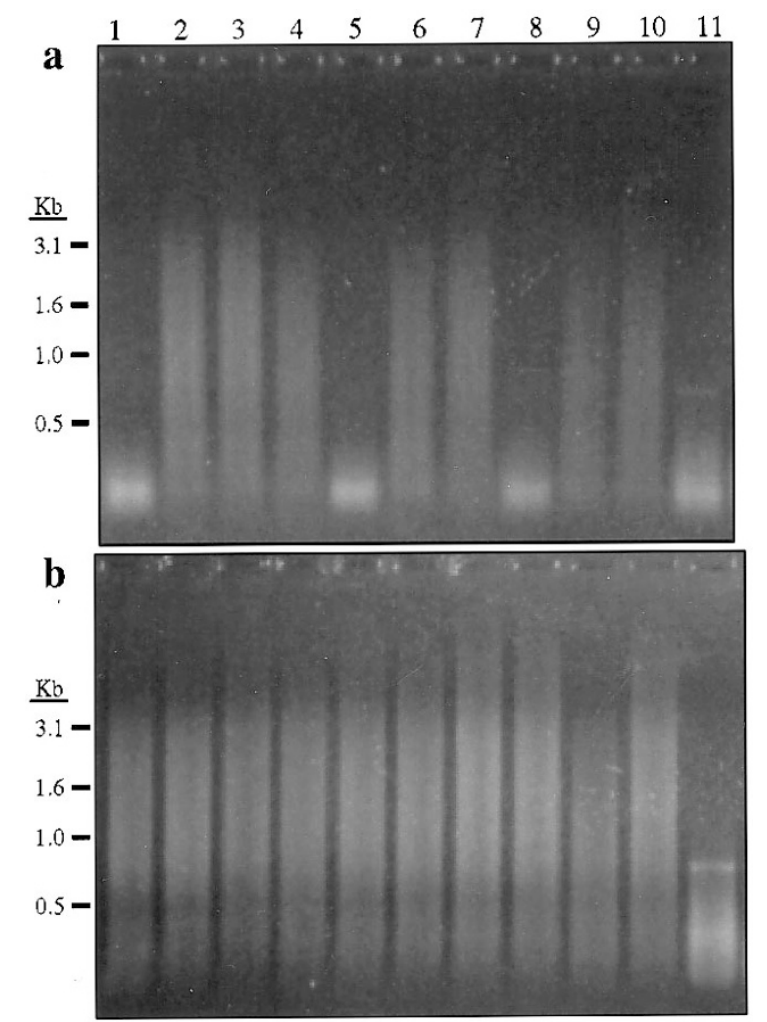

Fig. 2 PCR detection of paternal sex ratio (PSR) in Nasonia giraulti and N. longicornis. (a) An example of PCR amplification of DNA from individual N. giraulti males resulting from all-son broods (lanes 1-10) and a male from a mostly daughter brood (lane 11). Similar to PCR of N. vitripennis (Dobson \& Tanouye, 1996), a high molecular weight smear $(<4 \mathrm{~kb})$ is indicative of the presence of PSR. This smear is absent from the control male (lane 11). As previously discussed (Dobson \& Tanouye, 1996), this unusual amplification product (i.e. smearing pattern instead of a distinct band) may be caused by amplification within a highly repetitive region on PSR (Nur et al., 1988) and the subsequent concatamerization of the resulting multiple repeat products. However, for the diagnostic purposes of this study, these amplification products allow the distinguishing of wasps which harbour PSR. Also similar to N. vitripennis results, non-PSR males (lanes 1, 5, 8 and 11) show differing intensities of a low molecular weight band $(0.7 \mathrm{~kb})$ which may reflect amplification of sequences on the $N$. giraulti autosomes. In this experiment, seven of the 10 males from all-son broods were interpreted as harbouring PSR. The remaining three males from all-son broods (lanes 1, 5 and 8 ) and the control male (lane 11) are interpreted as non-PSR males resulting from unfertilized eggs. (b) An example of amplification of individual $N$. longicornis males from all-son broods (lanes 1-10) and a male from a mostly daughter brood (lane 11). Amplification products are similar to those observed in $N$. vitripennis and $N$. giraulti. All 10 of the males resulting from all-son broods (lanes 1-10) are interpreted as harbouring PSR. The control male (lane 11) is interpreted as a non-PSR male resulting from an unfertilized egg. 


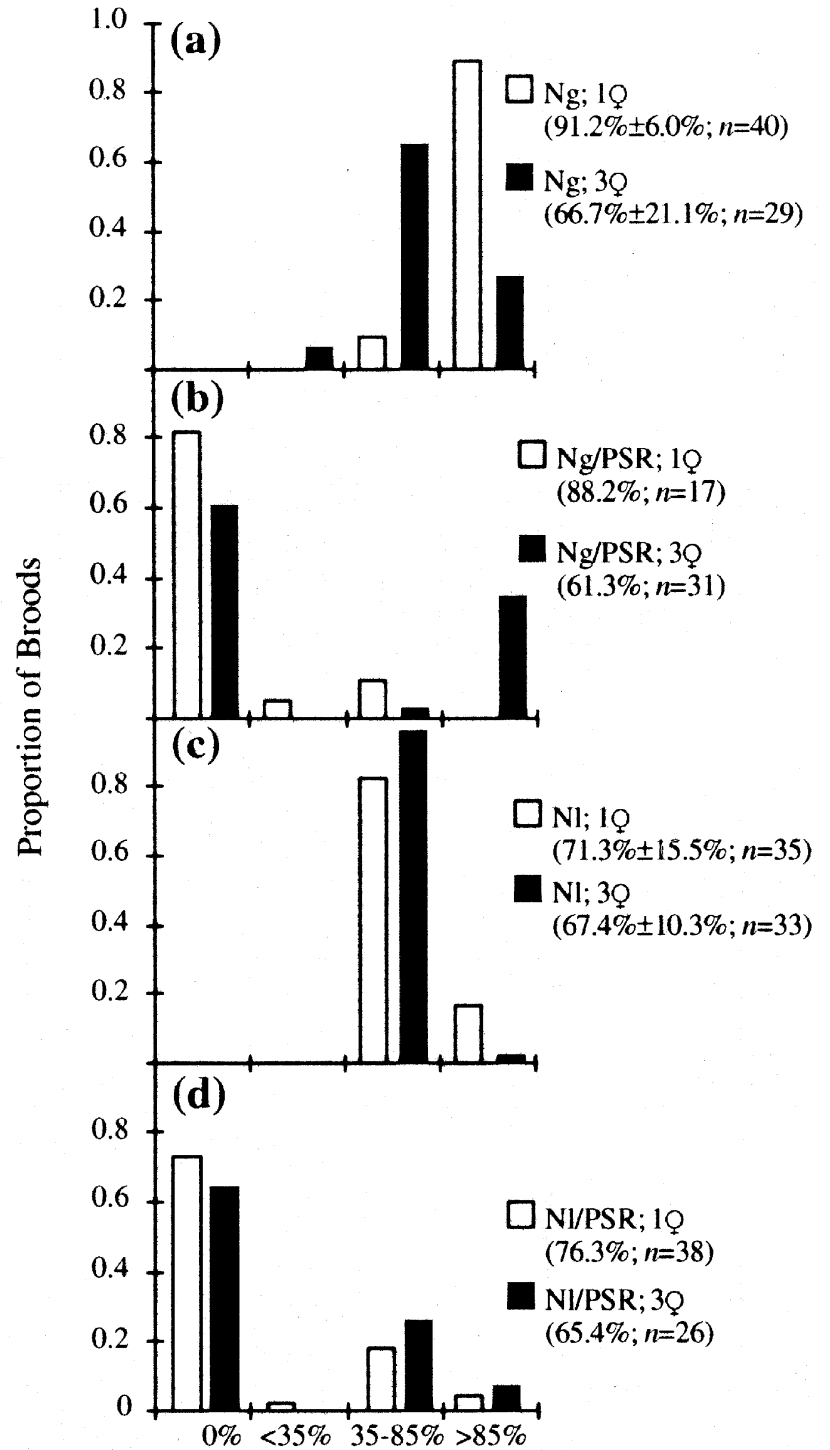

Brood sex ratio (\% Female)

Fig. 3 Histogram of the brood sex ratios resulting from crosses. Shown are the results of pair and group mated (a) non-PSR (paternal sex ratio chromosome) and (b) PSR crosses of Nasonia giraulti and pair and group mated (c) non-PSR and (d) PSR crosses of $N$. longicornis. Broods are divided into four categories depending on their brood sex ratios (percentage of daughters). For non-PSR crosses, the average brood sex ratio, standard deviation and number of broods examined are indicated in the legends. For PSR crosses, the percentage of PSR males present in broods and the number of broods examined are indicated in the legends. ' $\mathrm{Ng}$ ' indicates $N$. giraulti crosses, and ' $\mathrm{Nl}$ ' indicates $N$. longicornis crosses. ' 19 ' and ' 3 ' ' indicate pair mated and group mated crosses, respectively. 'PSR' indicates PSR crosses. to harbour PSR and produce all-son broods. Crossing results (Fig. 3) show that $88.2 \%$ and $76.3 \%$ of $N$. giraulti and $N$. longicornis sons resulting from PSR pair matings harbour PSR. This similarity indicates that in $N$. giraulti and $N$. longicornis, the transmission rate of PSR is equal to the fertilization rate, that the ability of PSR to induce chromosome loss was not impaired relative to $N$. vitripennis and that fertilized eggs are being converted from females to males harbouring PSR. Also similar to PSR within N. vitripennis, occasional PSR transmission failure may occur with $N$. giraulti and N. longicornis. Previous experiments have shown that $N$. vitripennis PSR males fail to transmit PSR in $\approx 10 \%$ of crosses (Werren \& van den Assem, 1986; Beukeboom \& Werren, 1993b). As shown in Fig. 3, one pair mating of $N$. giraulti and $N$. longicornis resulted in broods with rare daughters (2 daughters : 43 sons in $N$. giraulti, and 2 daughters: 51 sons in N. longicornis). This suggests that the fathers in these crosses harboured PSR but failed to transmit it to two of their offspring, resulting in daughters. However, confirmation of PSR transmission failure and the determination of its frequency in $N$. giraulti and $N$. longicornis will require additional analysis.

To verify the correlation between fertilization rate and the transmission rate of PSR in N. giraulti and $N$. longicornis, control and test crosses were conducted with group matings of a male with three females. Previous work with N. vitripennis has demonstrated that females fertilize a lower percentage of their eggs when crowded with other females (Werren, 1980, 1983, 1987a). Similar to N. vitripennis, this test demonstrated that $N$. giraulti females significantly $(P<0.001 ; t$-test $)$ lower their fertilization rate to $66.7 \%$ when grouped on hosts (Fig. 3). Corresponding to this reduction in the fertilization rate, transmission of PSR in $N$. giraulti showed a proportional reduction to $61.3 \%$ with grouped females. Nasonia longicornis females were different from $N$. vitripennis and $N$. giraulti in that they showed only a slight, nonsignificant $(P=0.19)$ decrease in the fertilization rate when crowded. Correlating to this small reduction in the fertilization rate, transmission of PSR through grouped N. longicornis females also showed a similar, small reduction relative to isolated females (Fig. 3). These crosses of PSR $N$. giraulti and $N$. longicornis males with conspecific females were continued for four generations. In all generations, PSR test crosses produced primarily all-son broods, whereas control crosses continued to produce primarily daughters. Similar to the PCR results, this demonstrates that PSR is stably transmitted at high rates (equivalent to fertilization) in 
both $N$. giraulti and $N$. longicornis. In addition, these crosses show that the ability of PSR to induce autosome loss is not significantly altered between the differing host backgrounds of all three species. The high sex ratio $(>85 \%)$ observed in group mated PSR N.giraulti broods which produce daughters (Fig. 3b) reflects the pair mated $F_{1}$ crosses as described previously.

Crossing experiments also suggest that the entire autosomal complement is lost in crosses of $N$. giraulti and $N$. longicornis PSR males. The partial loss of a chromosome complement would be expected to result in an increased incidence of aneuploidy and a reduced brood size. A comparison of brood sizes resulting from pair matings demonstrates that PSR harboured within $N$. giraulti and $N$. longicornis males does not significantly $(P=0.46$ and 0.29 , respectively; $t$-test) affect the size of broods relative to non-PSR control crosses (Table 1). This is similar to observations of PSR harboured within $N$. vitripennis males (Werren \& van den Assem, 1986; Beukeboom \& Werren, 1993b).

\section{Discussion}

These results demonstrate that PSR survives at high rates in interspecific crosses between $N$. vitripennis, $N$. giraulti and $N$. longicornis. They also show that transmission of PSR and its ability to induce paternal autosome loss are not altered in the different host backgrounds. In all three species, PSR is transmitted into fertilized eggs at rates equal to fertilization. One complement of autosomes is lost from these eggs, which subsequently develop as haploid males harbouring PSR. Based on the behaviour of PSR in N. vitripennis, the paternal complement is assumed to be lost in $N$. giraulti and $N$. longicornis PSR crosses. Alternatively, the maternal complement, or a mixture of paternal and maternal autosomes constituting one complement, could be lost in

Table 1 Comparison of pair mated Nasonia giraulti $(\mathrm{Ng})$ and $N$. longicornis $(\mathrm{Nl})$ brood sizes. Crosses of males harbouring the paternal sex ratio chromosome (PSR) are indicated adjacent to the species genotype. Brood size is shown as an average and standard deviation of the number of individuals per brood. $n=$ number of broods

\begin{tabular}{lcc}
\hline & $(\Downarrow \pm \mathrm{SD})$ & $(n)$ \\
\hline $\mathrm{Ng}$ & $61.0 \pm 15.7$ & 40 \\
$\mathrm{Ng} / \mathrm{PSR}$ & $64.6 \pm 24.7$ & 36 \\
$\mathrm{Nl}$ & $46.8 \pm 17.1$ & 35 \\
$\mathrm{Nl} / \mathrm{PSR}$ & $50.5 \pm 19.9$ & 58 \\
\hline
\end{tabular}

these species. However, the similarity of the behaviour of PSR in all three Nasonia species suggests that its mechanism of chromosomal loss does not differ from that observed in N. vitripennis (i.e. PSR is unlikely to switch from inducing paternal chromosome loss to inducing maternal chromosome loss). Confirmation of this hypothesis will require the future generation of chromosomal markers for $N$. giraulti and N. longicornis.

In previous studies of the Nasonia/PSR complex, loci on PSR have been identified which are homologous with autosomal sequences of $N$. giraulti and $N$. longicornis but do not hybridize with $N$. vitripennis autosomes (Eickbush et al., 1992; McAllister, 1995). This has been used as support for a hypothesis that PSR may have originated outside $N$. vitripennis. According to this hypothesis, PSR originated in an interspecific hybridization between $N$. longicornis and N. vitripennis (McAllister, 1995). Cytoplasmic incompatibility resulted in fragmentation of the N. longicornis autosomes. One of these fragments acquired the ability to induce paternal chromosome loss and subsequently evolved into PSR. Alternatively, these sequence comparisons may suggest that PSR originated and evolved functionality (i.e. its mechanism of inducing autosome loss) prior to its introduction into N. vitripennis. This alternative hypothesis would be unlikely if PSR were unable to function outside $N$. vitripennis (i.e. this would suggest that the functionality of PSR evolved subsequent to its introduction into $N$. vitripennis). The results of the present study provide an initial test of this hypothesis and show that the mechanisms of PSR are not specific for N. vitripennis.

Interestingly, PSR may be ideally suited for interspecific transfer because it eliminates all of the autosomes which it accompanies. Progeny resulting from interspecific matings are typically less fecund because of negative epistatic interactions occurring between the nuclear genes of the two species (Breeuwer \& Werren, 1995). This hybrid breakdown is generally attributed to different coadapted gene complexes in the two species, which are broken up by recombination. The paternal sex ratio may circumvent this hybrid breakdown by eliminating the male's autosomal complement. The resulting progeny harbour only the maternal chromosomes and PSR with no opportunity for negative epistatic interactions. Although other B chromosomes do not cause paternal autosome loss, their meiotic drive systems may also facilitate their spread in the recipient species if transferred interspecifically. This potential for interspecific movement of other B chromosomes is supported by previously reported

(C) The Genetical Society of Great Britain, Heredity, 81, 261-269. 
sequence comparisons showing significant differences between other supernumerary chromosomes and the autosomes of their hosts (Beukeboom, 1994). Because hybrid breakdown is generally recessive (Breeuwer \& Werren, 1995), an interspecific mating followed by several generations of backcrosses with the recipient species could provide a means for other B chromosomes to be transferred interspecifically.

The potential for naturally occurring interspecific transfers is of ecological importance because these transfers can occur into both beneficial and pest species. Many of the closest relatives of Nasonia, and therefore the most likely candidates for natural transfers of PSR, are beneficial parasitoids. Because of the overproduction of males, the introduction of PSR would be expected to affect the population structure of the recipient host, causing extinction of the host in the most extreme examples. Unpublished field collections suggest that PSR transfer and its subsequent maintenance in the recipient species are rare events. These collections have failed to locate PSR in species other than N. vitripennis (McAllister, 1995). These results could reflect: (i) limited sampling; (ii) the rarity of naturally occurring interspecific transfers of PSR; and/or (iii) the difficulty in maintaining PSR in populations subsequent to its transfer.

The opportunity for naturally occurring interspecific transfers would be reduced by limited sympatry of the populations of 'donor' species, which harbour PSR, and 'recipient' species, which can potentially mate with the donor species. Mating preferences would further reduce the potential for interspecific matings; for example, although $N$. vitripennis and $N$. giraulti are sympatric in eastern North America (Darling \& Werren, 1990), comparisons of courtship behaviour suggest that mate discrimination may occur between these species (van den Assem \& Werren, 1994).

Cytoplasmic incompatibility would be expected to result in the postmating loss of PSR if the female were infected with a different Wolbachia strain (Beukeboom et al., 1992; Beukeboom \& Werren, 1993a). However, previous studies have demonstrated that older males show a lower incidence of cytoplasmic incompatibility (Giordano et al., 1995). This may provide a route for PSR transfers in nature. The paternal sex ratio chromosome has also been shown to survive cytoplasmically incompatible crosses at a low rate (Beukeboom et al., 1992; Beukeboom \& Werren, 1993a).

The paternal sex ratio chromosome might also be lost subsequent to an interspecific transfer if the recipient population were unable to maintain it. Previous models (Skinner, 1987; Werren, 1991; Beukeboom \& Werren, 1992; Werren \& Beukeboom, 1993) demonstrate relatively narrow ranges of conditions which must be satisfied for the maintenance of PSR within a population. Populations outside these ranges would be expected to lose PSR or to become extinct; for example, highly subdivided populations or populations with low fertilization rates would be expected to lose PSR. Alternatively, if PSR were introduced into a panmictic population with high fertilization rates, PSR could drive this population to extinction. However, in these latter examples, PSR could be transferred into additional species prior to PSR loss or population extinction.

As an example of a possible interspecific transfer which may have already occurred, PSR has been shown to share sequence homology with Trichomalopsis dubius (Eickbush et al., 1992). This sequence homology between PSR and T.dubius probably reflects the occurrence of PSR prior to the divergence of the Trichomalopsis and Nasonia genera (Eickbush et al., 1992). However, an alternative hypothesis might be that PSR crossed the species barrier between T.dubius and $N$. vitripennis more recently, in a step-wise fashion. In this scenario, movement of PSR between these reproductively isolated species could be accomplished via sequential, interspecific transfers between species which are capable of mating. The paternal sex ratio chromosome could be subsequently lost from all of these species (as described above) leaving only $N$. vitripennis harbouring PSR. This hypothetical scenario is intended only to demonstrate the potential for movement of PSR. Interestingly, results of the present study have demonstrated that this type of step-wise movement of PSR can serve to transfer PSR across species barriers. Specifically, it was possible to bypass the reproductive barrier between $N$. vitripennis and $N$. longicornis through a third species capable of mating with both (i.e. $N$. giraulti).

The ability to transfer PSR or its mechanisms interspecifically suggests its potential as a biological control method of economically important pests (e.g. Solenopsis invicta, fire ant). Results of this study demonstrate that PSR can be successfully transferred and can continue to induce paternal autosome loss in N.giraulti and N. longicornis. This suggests the potential for transfers into more distantly related species. Although serious technological hurdles must be overcome to accomplish these proposed transfers, the results presented here suggest that PSR may continue to function in additional species. 
Based on these results, future focuses should include developing techniques for transferring PSR into more distantly related species and an extensive search for naturally occurring transfers of PSR. Field-collected N. giraulti and N. longicornis males would be good initial candidates for this proposed search; but additional species, even those unable to mate directly with $N$. vitripennis, should also be examined. This search may answer questions concerning the origin of PSR and whether interspecific PSR movement occurs naturally. This search may also provide information about the limits of the host range for PSR; for example, one would expect PSR to be limited to haplodiploid species, because paternal autosome loss in diploid species is lethal. An example of another potential limitation might be the multiple mechanisms of sex determination found in Hymenoptera (Beukeboom 1995; Cook \& Crozier, 1995). If the mechanisms of transmission and autosome loss of PSR are linked to the sex determination mechanism of $N$. vitripennis, one would expect the mechanisms of PSR to be affected when transferred into species with a different mechanism of sex determination. Identification of these limitations may provide important clues about the mechanisms of transmission and paternal autosome loss of PSR.

\section{Acknow ledgements}

We would like to thank John Werren for supplying wasp strains and species needed for this work. We also appreciate his and Bryant McAllister's useful discussions of PSR/autosome homologies and the potential origin of PSR. We thank Scott O'Neill, Jeremy Lee and Elaine Reynolds and two anonymous reviewers for their critical reading of this manuscript and their suggestions. This work was supported in part by grants from the National Institutes of Health (EY/NS10824) and the U.C. Berkeley Agricultural Experiment Station.

\section{References}

VAN DEN ASSEM, J. 1977. A note on the ability to fertilize following insemination (with females of Nasonia vitripennis, Hymenoptera: Chalcidoidea). Neth. J. Zool., 27, 230-235.

VAN DEN ASSEM, J. AND WERREN, J. H. 1994. A comparison of the courtship and mating behavior of three species of Nasonia (Hymenoptera, Pteromalidae). J. Insect Behav., 7, 53-66.

BEUKEBOOM, L. w. 1994. Bewildering Bs: an impression of the $1^{\text {st }}$ B-Chromosome Conference. Heredity, 73,
$328-336$.

Beukeboom, L. w. 1995. Sex determination in Hymenoptera-a need for genetic and molecular studies. Bioessays, 17, 813-817.

Beukeboom, L. W. AND Werren, J. H. 1992. Population genetics of a parasitic chromosome: experimental analysis of PSR in subdivided populations. Evolution, 46, 1257-1268.

BEUKEBOOM, L. W. AND WERREN, J. H. 1993a. Deletion analysis of the selfish B chromosome, paternal sex ratio (PSR), in the parasitic wasp Nasonia vitripennis. Genetics, 133, 637-648.

BEUKeBOOM, L. W. AND WERREN, J. H. 1993b. Transmission and expression of the parasitic paternal sex ratio (PSR) chromosome. Heredity, 70, 437-443.

BEUKEBOOM, L. W., REED, K. M. AND WERREN, J. H. 1992. Effects of deletions on mitotic stability of the paternal sex ratio (PSR) chromosome from Nasonia. Chromosoma, 102, 20-26.

BREEUWER, J. A. AND WERREN, J. H. 1990. Microorganisms associated with chromosome destruction and reproductive isolation between two insect species. Nature, 346, $558-560$.

BREEUWER, J. A. AND WERREN, J. H. 1995. Hybrid breakdown between two haplodiploid species: the role of nuclear and cytoplasmic genes. Evolution, 49, 705-717.

COOK, J. M. AND CROZIER, R. H. 1995. Sex determination and population biology in the Hymenoptera. Trends Ecol. Evol., 10, 281-286.

DARLing, D. C. AND WERREN, J. H. 1990. Biosystematics of Nasonia (Hymenoptera: Pteromalidae): two new species reared from bird's nests in North America. Ann. Entomol. Soc. Am., 83, 352-370.

DOBSON, s. AND TANOUYE, M. 1996. The paternal sex ratio chromosome induces chromosome loss independently of Wolbachia in the wasp Nasonia vitripennis. Devel. Genes Evol., 206, 207-217.

EICKBUSH, D. G, EICKBUSH, T. H. AND WERREN, J. H. 1992. Molecular characterization of repetitive DNA sequences from a B chromosome. Chromosoma, 101, $575-583$.

GIORDANO, R., O'NEILl, S. L. AND ROBERTSON, H. M. 1995. Wolbachia infections and the expression of cytoplasmic incompatibility in Drosophila sechellia and D. mauritiana. Genetics, 140, 1307-1317.

JONES, R. N. 1991. B-chromosome drive. Am. Nat., 137, 430-442.

JONES, R. N. AND REES, H. 1982. B-chromosomes. Academic Press, London.

MCALLISTER, B. F. 1995. Isolation and characterization of a retroelement from $\mathrm{B}$ chromosome (PSR) in the parasitic wasp Nasonia vitripennis. Insect Mol. Biol., 4, 253-262.

NUR, U., WERREN, J. H., EICKBUSH, D. G., BURKE, W. D. AND EICKBUSH, T. H. 1988. A selfish' B chromosome that enhances its transmission by eliminating the paternal genome. Science, 240, 512-514.

RYAN, S. L., SAUL, G. B., II AND CONNER, G. W. 1987. Separ-

(C) The Genetical Society of Great Britain, Heredity, 81, 261-269. 
ation of factors containing R-locus genes in Mormoniella stocks derived from aberrant segregation following incompatible crosses. J. Hered., 78, 273-275.

SKINNER, s. w. 1987. Paternal transmission of an extrachromosomal factor in a wasp: evolutionary implications. Heredity, 59, 47-53.

WERREN, J. H. 1980. Sex ratio adaptations to local mate competition in a parasitic wasp. Science, 208, 1157-1160.

WERREN, J. H. 1983. Sex ratio evolution under local mate competition in a parasitic wasp. Evolution, 37, 116-124.

WERREN, J. H. 1987a. The coevolution of autosomal and cytoplasmic sex ratio factors. J. Theor. Biol., 124, 317-334.

WERrEN, J. H. 1987b. Labile sex ratios in wasps and bees.
Bioscience, 37, 498-506.

WERREN, J. H. 1991. The paternal sex ratio chromosome of Nasonia. Am. Nat., 137, 392-402.

WERREN, J. H. AND VAN DEN ASSEM, J. 1986. Experimental analysis of a paternally inherited extrachromosomal factor. Genetics, 114, 217-233.

Werren, J. H. AND BeUKeboom, L. W. 1993. Population genetics of a parasitic chromosome: theoretical analysis of PSR in subdivided populations. Am. Nat., 142, 224-241.

WERREN, J. H., NUR, U. AND EICKBUSH, D. 1987. An extrachromosomal factor causing loss of paternal chromosomes. Nature, 327, 75-76.

WHITING, A. R. 1967. The biology of the parasitic wasp, Mormoniella vitripennis. Q. Rev. Biol., 42, 334-406. 